PROCEEDINGS OF THE

AMERICAN MATHEMATICAL SOCIETY

Volume 130, Number 12, Pages 3473-3481

S 0002-9939(02)06570-X

Article electronically published on April 22, 2002

\title{
ON THE DIMENSION OF THE SPACE OF THETA FUNCTIONS
}

\author{
DANIEL BUMP AND ALEXANDER PEKKER \\ (Communicated by Dennis A. Hejhal)
}

\begin{abstract}
We compute the dimension of the space of theta functions of a given type using a variant of the Selberg trace formula.
\end{abstract}

\section{Main Result}

Theta functions play an important role in the theory of abelian varieties; for instance, theta functions are used to construct meromorphic functions on a multidimensional torus and to embed a multidimensional torus into projective space (see, for instance, 8, [10, 14]).

Frobenius 4 computed the dimensions of spaces of theta functions in several variables. As far as we know, other expositions of this theorem ([8], 7, 14) more or less follow Frobenius in making Fourier expansions and discussing the relations between the Fourier coefficients.

Selberg [12] and Eichler [3] invented a method of computing the dimensions of spaces of modular forms which has proved important. One constructs an automorphic reproducing kernel by averaging the Bergman kernel function, then computes the dimension of a space of modular forms as the trace of the kernel. See [13] for an accessible account of this technique, and Hejhal [5] for a recent variation.

In these examples, the Bergman kernel function is a function on a Hermitian symmetric space, conformally equivalent to a bounded domain in $\mathbb{C}^{n}$. In a different domain- $\mathbb{C}^{n}$ itself-Bargman [1] and independently Newman and Shapiro [9] introduced a Bergman kernel function. This is a reproducing kernel for the Fock space, a Hilbert space of entire functions. The relevance of the Fock space and the reproducing aspect of this kernel to the theory of theta functions was revealed by Cartier [2] and Satake [11] and (implicitly, earlier) by Weil [15]. See also Hurt [6], Chapter 8 .

We imitate Eichler and Selberg in constructing an automorphic reproducing kernel for theta functions by averaging the Bergman kernel function on the Fock space, and we recover Frobenius' dimension formula by computing the trace of this reproducing kernel.

Let $\Lambda$ be a lattice in $\mathbb{C}^{n}$ (i.e., $\Lambda$ is a $\mathbb{Z}$-module of dimension $2 n$ ). A theta function of $(L, M)$ type on $\mathbb{C}^{n}$ with respect to $\Lambda$ is a meromorphic function, not identically

Received by the editors July 12, 2001.

2000 Mathematics Subject Classification. Primary 14K25.

We would like to thank Dennis Hejhal for help with the references.

(C)2002 American Mathematical Society 
zero, satisfying

$$
\theta(z+\lambda)=\theta(z) J(z \lambda)=\theta(z) \exp [2 \pi i(L(z, \lambda)+M(\lambda))]
$$

for every $z \in \mathbb{C}^{n}$ and $\lambda \in \Lambda$, where $L(z, \lambda)$ is linear in $z$. It is easily checked that all entire theta function of a given $(L, M)$ type together with the zero function form a complex vector space, which we denote by $\mathcal{T}_{L, M}$. Further, define

$$
E(z, w)=L(z, w)-L(w, z) \text { and } H(z, w)=E(i z, w)+i E(z, w) .
$$

Classical results show that $E$ is a Riemann form and $H$ is a positive Hermitian form. Moreover, $H$ is positive definite if and only if $E$ is nondegenerate. If we select a $\mathbb{Z}$-basis for $\Lambda$, then it is also a basis for $\mathbb{C}^{n}$, considered as a real vector space of dimension $2 n$. In such a basis, the $\sqrt{\operatorname{det} E}$ is an integer independent of the choice of $\mathbb{Z}$-basis for $\Lambda$ and is called the Pfaffian of $E$.

Theorem 1.1 (Frobenius). Let $\Lambda$ be a lattice in $\mathbb{C}^{n}$, and let $(L, M)$ be a type of theta functions. Let $E$ and $H$ be as in (1.2). Supposing that $E$ is nondegenerate, let $P f$ denote the Pfaffian of $E$. Then

$$
\operatorname{dim} \mathcal{T}_{L, M}=\operatorname{det}(H) \operatorname{Vol}\left(\mathbb{C}^{n} / \Lambda\right)=P f .
$$

When $E$ is degenerate, classical results (see Lang [8, pp. 89-90]) show that $W=\operatorname{ker}(E)=\operatorname{ker}(H)$ is a subspace of $\mathbb{C}^{n}$. Let $\Lambda_{0}$ denote the image of $\Lambda$ in $\mathbb{C}^{n} / W$, $\left(L_{0}, M_{0}\right)$ the induced type on $\mathbb{C}^{n} / W$, and $E_{0}$ and $H_{0}$ the induced forms on $\mathbb{C}^{n} / W$. It is easy to show that $\Lambda_{0}$ is a lattice, $E_{0}$ is nondegenerate, and $H_{0}$ is positive definite. Again, picking a $\mathbb{Z}$-basis for $\Lambda_{0}, \sqrt{\operatorname{det} E_{0}}$ is an integer, called the reduced Pfaffian of $E$. It is not hard to see that $\operatorname{dim} \mathcal{T}_{L, M}=\operatorname{dim} \mathcal{T}_{L_{0}, M_{0}}$, so Theorem 1.1 implies the following stronger result.

Theorem 1.2. Let $\Lambda$ be a lattice in $\mathbb{C}^{n}$, let $(L, M)$ be a type of theta functions, and let $E$ and $H$ be as in (1.2). Let $W$ denote $\operatorname{ker}(E), \Lambda_{0}$ the image of $\Lambda$ in $\mathbb{C}^{n} / W$, $H_{0}$ the reduced form of $H$, and $P f_{\mathrm{RED}}$ the reduced Pfaffian of $E$. Then

$$
\operatorname{dim} \mathcal{T}_{L, M}=\operatorname{det}\left(H_{0}\right) \operatorname{Vol}\left((\mathbb{C} / W) / \Lambda_{0}\right)=P f_{\mathrm{RED}}
$$

The proof of Theorem 1.1 follows immediately from the first observation in Section 5 and Theorems 5.2 and 5.3 .

\section{Properties of the Reproducing Kernel}

A reproducing kernel in a finite-dimensional Hilbert space $\mathcal{H} \subset \mathrm{L}^{2}(X, \mu)$ is the function $\Phi: X \times X \rightarrow \mathbb{C}$ satisfying the following three properties:

1. For fixed $w, f(z)=\Phi(z, w) \in \mathcal{H}$.

2. For every $f \in \mathcal{H}, f(z)=\int_{X} \Phi(z, w) f(w) d \mu(w)$.

3. $\Phi(z, w)=\overline{\Phi(w, z)}$.

If $\mathcal{H}$ is a finite-dimensional vector space, then integrating the reproducing kernel for $\mathcal{H}$ "along the diagonal" yields the dimension of $\mathcal{H}$.

Theorem 2.1. Let $\mathcal{H} \subset L^{2}(X, \mu)$ be a finite-dimensional Hilbert space. Then there exists a unique reproducing kernel $\Phi$ and

$$
\operatorname{dim} \mathcal{H}=\int_{X} \Phi(z, z) d \mu(z) .
$$


Proof. Let $\left\{\phi_{1}, \ldots, \phi_{n}\right\}$ be an orthonormal basis for $\mathcal{H}$. Then the kernel $\Phi(z, w)=$ $\sum_{i=1}^{n} \phi_{i}(z) \overline{\phi_{i}(w)}$ is the unique reproducing kernel for $\mathcal{H}$ and integrating along the diagonal clearly gives $n$.

\section{Properties of theta Functions}

In this section we collect several results from Lang [8, pp. 83-89]. Recall that a theta function on $\mathbb{C}^{n}$ with respect to a lattice $\Lambda$ is a meromorphic function, not identically zero, satisfying (1.1). The theta multiplier, $J$, satisfies the cocycle identity

$$
J(z, \lambda+\nu)=J(z, \lambda) J(z+\lambda, \nu) .
$$

Theorem 3.1. Let $\theta$ be a theta function of $(L, M)$ type, and define $K(z)=M(z)-$ $\frac{1}{2} L(z, z)$. Then $\theta$ satisfies

$$
\theta(z+\lambda)=\theta(z) J(z, \lambda)=\theta(z) \exp \left[2 \pi i\left(L(z, \lambda)+\frac{1}{2} L(\lambda, \lambda)+K(\lambda)\right)\right]
$$

where $L$ is defined on $\mathbb{C}^{n} \times \mathbb{C}^{n}$ and is $\mathbb{C}$-linear in the first component and $\mathbb{R}$-linear in the second component, and $K$ is defined on $\mathbb{C}^{n}$ and is $\mathbb{R}$-linear.

Let $\theta$ be a theta function as in Theorem 3.1, and define $E$ and $H$ as in (1.2).

Theorem 3.2. $E$ is alternating and real-valued for all $z, w \in \mathbb{C}^{n}$ and integervalued on all $z, w \in \Lambda$. Moreover, the form $q(z, w)=E(i z, w)$ is symmetric. (In other words, $E$ is a Riemann form.) The form $H$ is Hermitian and positive, and $E(z, w)=\operatorname{Im} H(z, w)$.

A theta function $\theta$ is a normalized theta function if it satisfies (3.2) and $H(z, w)=$ $2 i L(z, w)$ and $K(z) \in \mathbb{R}$ for all $z \in \mathbb{C}^{n}$. In this case relation (3.2) can be written in terms of $H$ and $K$ as

$$
\theta(z+\lambda)=\theta(z) \exp \left[\pi H(z, \lambda)+\frac{\pi}{2} H(\lambda, \lambda)+2 \pi i K(\lambda)\right]
$$

and the theta multiplier $J$ becomes

$$
J(z, \lambda)=\exp \left[\pi H(z, \lambda)+\frac{\pi}{2} H(\lambda, \lambda)+2 \pi i K(\lambda)\right] .
$$

Normalized entire theta functions satisfy the following growth condition.

Theorem 3.3. If $\theta$ is a normalized entire theta function with associated Hermitian form $H$, then there exists a constant $C$ such that $|\theta(z)|^{2} e^{-\pi H(z, z)}<C$.

For normalized entire theta functions we will denote the type by $(H, K)$ instead of $(L, M)$ and the associated vector space as $\mathcal{N}_{H, K}$. Finally, we have the following isomorphism theorem.

Theorem 3.4. Let $\theta(z)$ be an entire theta function of $(L, M)$ type. Then there exists a unique normalized entire theta function $\theta_{N}(z)$ of $(H, K)$ type and a trivial theta function $\theta_{T}(z)$ such that $\theta_{N}(z)=\theta(z) \theta_{T}(z)$, up to multiplication by a constant. Moreover, $\operatorname{dim} \mathcal{T}_{L, M}=\operatorname{dim} \mathcal{N}_{H, K}$. 


\section{Auxiliary space}

Let $H$ be an $n \times n$ positive definite Hermitian form. Then, $H(z, w)=w^{*} H z$, where the second $H$ is a Hermitian matrix. By the spectral theorem, there exists a unitary matrix $U$ and a diagonal matrix $D$ such that

$$
U^{*} H U=D=\left[\begin{array}{cccc}
\Delta_{1} & 0 & \ldots & 0 \\
0 & \Delta_{2} & \ldots & 0 \\
\vdots & \vdots & \ddots & 0 \\
0 & 0 & \ldots & \Delta_{n}
\end{array}\right]
$$

and all the $\Delta_{i}$ are real and positive. Moreover, $\Delta=\prod_{i=1}^{n} \Delta_{i}=\operatorname{det}(H)$.

Define the measure $\mu$ as $d \mu(z)=\Delta e^{-\pi H(z, z)} d z$ (where $d z$ is Lebesgue measure), and let $\mathcal{H}_{H}$ be the space of all analytic functions $f: \mathbb{C}^{n} \rightarrow \mathbb{C}$ for which

$$
\int_{\mathbb{C}^{n}}|f(z)|^{2} d \mu(z)=\int_{\mathbb{C}^{n}}|f(z)|^{2} \Delta e^{-\pi H(z, z)} d z<\infty .
$$

It is clear that $\mathcal{H}_{H}$ is a subset of $L^{2}(\mu)$, and is therefore a pre-Hilbert space with the inner product

$$
\left(f_{1}, f_{2}\right)=\int_{\mathbb{C}^{n}} f_{1}(z) \overline{f_{2}(z)} d \mu(z) .
$$

Following Igusa [7, pp. 31-33], we will show that it is in fact a Hilbert space.

Theorem 4.1. Let

$$
\phi_{\left(p_{1}, \ldots, p_{n}\right)}(z)=\prod_{i=1}^{n} \sqrt{\frac{\left(\pi \Delta_{i}\right)^{p_{i}}}{p_{i} !}}\left(U^{-1} z\right)_{i}^{p_{i}}
$$

where $\left(p_{1}, \ldots, p_{n}\right)$ range over all $n$-tuples of nonnegative integers. Then $\left\{\phi_{\left(p_{1}, \ldots, p_{n}\right)}\right\}$ is an orthonormal set in $\mathcal{H}_{H}$.

Proof. Making the substitution $w=U^{-1} z$, the theorem reduces to the one-dimensional case where it can be easily checked.

Lemma 4.2. Let $\left\{\phi_{\left(p_{1}, \ldots, p_{n}\right)}\right\}$ be as in Theorem 4.1. Then

$$
\sum_{\left(p_{1}, \ldots, p_{n}\right)} \phi_{\left(p_{1}, \ldots, p_{n}\right)}(z) \overline{\phi_{\left(p_{1}, \ldots, p_{n}\right)}(w)}=e^{\pi H(z, w)} .
$$

Proof. Since $\left(p_{1}, \ldots, p_{n}\right)$ range over all $n$-tuples of nonnegative integers, the summation becomes

$$
\begin{aligned}
\sum_{\left(p_{1}, \ldots, p_{n}\right)} \phi_{\left(p_{1}, \ldots, p_{n}\right)}(z) \overline{\phi_{\left(p_{1}, \ldots, p_{n}\right)}(w)} & =\sum_{\left(p_{1}, \ldots, p_{n}\right)} \prod_{i=1}^{n} \frac{\left(\pi \Delta_{i}\right)^{p_{i}}}{p_{i} !}\left(U^{-1} z\right)_{i}^{p_{i}} \overline{\left(U^{-1} w\right)_{i}^{p_{i}}} \\
& =\prod_{i=1}^{n}\left(\sum_{j=0}^{\infty} \frac{\left(\pi \Delta_{i}\right)^{j}}{j !}\left(U^{-1} z_{i}\right)^{j} \overline{\left(U^{-1} w_{i}\right)^{j}}\right) \\
& =\prod_{i=1}^{n} e^{\pi \Delta_{i}\left(U^{-1} z\right)_{i} \overline{\left(U^{-1} w\right)_{i}}} .
\end{aligned}
$$

Since $H$ is Hermitian, $\sum_{i=1}^{n}\left(w^{*} U\right)_{i} \Delta_{i}\left(U^{*} z\right)_{i}=w^{*} U D U^{*}=w^{*} H^{*} z=w^{*} H z$, so the proof is complete. 
The following lemma bounds the value of $|f(z)|$ in terms of $\|f\|$; this estimate is helpful in relating uniform convergence on compact sets to $L^{2}(\mu)$ convergence.

Lemma 4.3. For any $f \in \mathcal{H}_{H}$ and any $z \in \mathbb{C}^{n},|f(z)| \leq e^{\frac{\pi}{2} H(z, z)}\|f\|$.

Proof. Since $f(U z)$ is analytic, by making the substitution $z \rightarrow U^{-1} z, f$ has a power series expansion which may be written as

$$
f(z)=\sum_{\left(p_{1}, \ldots, p_{n}\right)} c_{\left(p_{1}, \ldots, p_{n}\right)} \phi_{\left(p_{1}, \ldots, p_{n}\right)}(z),
$$

for some suitable constants $c_{\left(p_{1}, \ldots, p_{n}\right)}$ and orthonormal functions $\phi_{\left(p_{1}, \ldots, p_{n}\right)}$ as in Theorem 4.1. Since $f \in \mathcal{H}_{H}$,

$$
\sum_{\left(p_{1}, \ldots, p_{n}\right)}\left|c_{\left(p_{1}, \ldots, p_{n}\right)}\right|^{2}=\|f\|^{2}<\infty .
$$

By the Cauchy-Schwarz Inequality and Lemma 4.2,

$$
\begin{aligned}
|f(z)| & =\left|\sum_{\left(p_{1}, \ldots, p_{n}\right)} c_{\left(p_{1}, \ldots, p_{n}\right)} \phi_{\left(p_{1}, \ldots, p_{n}\right)}(z)\right| \\
& \leq \sqrt{\sum_{\left(p_{1}, \ldots, p_{n}\right)}\left|c_{\left(p_{1}, \ldots, p_{n}\right)}\right|^{2}} \sqrt{\sum_{\left(p_{1}, \ldots, p_{n}\right)}\left|\phi_{\left(p_{1}, \ldots, p_{n}\right)}(z)\right|^{2}}=e^{\frac{\pi}{2} H(z, z)}\|f\| .
\end{aligned}
$$

Theorem 4.4. $\mathcal{H}_{H}$ is a Hilbert space.

Proof. The only property we must show is completeness. By definition $\mathcal{H}_{H} \subset L^{2}(\mu)$ with the $\|\cdot\|$ norm being the $L^{2}(\mu)$ norm. So, suppose that $\left\{f_{i}\right\}$ is a Cauchy sequence in $\mathcal{H}_{H}$; we must show that its limit is also in $\mathcal{H}_{H}$. Since $\mathcal{H}_{H} \subset L^{2}(\mu)$, $\left\{f_{i}\right\}$ is Cauchy in $L^{2}(\mu)$, there exists a function $f \in L^{2}(\mu)$ such that $f_{i} \rightarrow f$ in the $\|\cdot\|$ norm.

On the other hand, since $\left\{f_{i}\right\}$ is Cauchy and since we can bound $e^{\frac{\pi}{2} H(z, z)}$ on any compact set $B$, Lemma 4.3 implies that the sequence $\left\{f_{i}\right\}$ is uniformly Cauchy on compact sets. This means that $f_{i}$ converges to some analytic function $g$ pointwise and uniformly on compact sets.

Thus if we can show that $f=g$, we will have that $f_{i} \rightarrow f$ in the $\|\cdot\|$ norm, with $f$ analytic and $\|f\|<\infty$. In other words, we will have that $f \in \mathcal{H}_{H}$.

Suppose on the contrary that $f \neq g$. If $B \subset \mathbb{C}^{n}$ is compact, define $\|\cdot\|_{B}$ by $\|h\|_{B}^{2}=\int_{B}|h(z)|^{2} d \mu(z)$. It is easy to see that $\|\cdot\|_{B}$ is a norm and that $\|h\|_{B} \leq$ $\|h\|$ for any function $h$. Assuming $f \neq g,\|f-g\|_{B}>\delta$ for some compact set $B$ and $\delta>0$. Since $f_{i} \rightarrow g$ uniformly on the compact set $B$, for $l$ sufficiently large, $\left\|g-f_{l}\right\|_{B}<\delta / 2$. On the other hand, since $f_{i} \rightarrow f$ in the $\|\cdot\|$ norm, for $k$ sufficiently large, $\left\|f-f_{k}\right\|_{B}<\delta / 2$. Taking $m=\max (k, l)$, the triangle inequality yields $\|f-g\|_{B}<\delta$, which is a contradiction.

The Hilbert space $\mathcal{H}_{H}$ is the Fock space ([1], [11, 7]). We have shown that the set defined in Theorem 4.1 is orthonormal. The next theorem shows that it is also complete. Having found a complete, orthonormal set, we will be ready to construct a reproducing kernel for $\mathcal{H}_{H}$.

Theorem 4.5. The orthonormal system $\left\{\phi_{\left(p_{1}, \ldots, p_{n}\right)}\right\}$ defined in Theorem 4.1 is complete. 
Proof. We must show that for any $f \in \mathcal{H}_{H}$, the partial sums

$$
\sum_{p_{1}, \ldots, p_{n}<N}\left(f, \phi_{\left(p_{1}, \ldots, p_{n}\right)}\right) \phi_{\left(p_{1}, \ldots, p_{n}\right)}
$$

converge to $f$ in the $\|\cdot\|$ norm.

Since $f$ is analytic, it has a power series expansion (4.1). Then, since $\left\{\phi_{\left(p_{1}, \ldots, p_{n}\right)}\right\}$ form an orthonormal set, we have $\left(f, \phi_{\left(p_{1}, \ldots, p_{n}\right)}\right)=c_{\left(p_{1}, \ldots, p_{n}\right)}$. By Bessel's Equality and (4.2),

$$
\begin{array}{r}
\left\|f-\sum_{p_{1}, \ldots, p_{n}<N}\left(f, \phi_{\left(p_{1}, \ldots, p_{n}\right)}\right) \phi_{\left(p_{1}, \ldots, p_{n}\right)}\right\|^{2}=\|f\|^{2}-\sum_{p_{1}, \ldots, p_{n}<N}\left|\left(f, \phi_{\left(p_{1}, \ldots, p_{n}\right)}\right)\right|^{2} \\
=\sum_{\left(p_{1}, \ldots, p_{n}\right)}\left|c_{\left(p_{1}, \ldots, p_{n}\right)}\right|^{2}-\sum_{p_{1}, \ldots, p_{n}<N}\left|c_{\left(p_{1}, \ldots, p_{n}\right)}\right|^{2} .
\end{array}
$$

As $N \rightarrow \infty$, the expression on the right converges to 0 , completing the proof.

Theorem 4.6. Let $\Phi(z, w)=e^{\pi H(z, w)}$. Then $\Phi$ is the reproducing kernel for $\mathcal{H}_{H}$.

Proof. In light of Lemma 4.2 and Theorem 4.5 it is clear that $\Phi$ satisfies all three properties of being a reproducing kernel for $\mathcal{H}_{H}$.

\section{Proof of Theorem 1.1}

In light of Theorem 3.4 it is sufficient to prove Theorem 1.1 only in the case of normalized entire theta functions, i.e., for the space $\mathcal{N}_{H, K}$ where $H$ is positive definite. Given this fact, the proof follows immediately from Theorem 5.2 and 5.3 The first of these theorems is proved by constructing a reproducing kernel for $\mathcal{N}_{H, K}$; the second follows by direct calculation.

Throughout this section, let $H$ and $K$ be fixed. Define the inner product on $\mathcal{N}_{H, K}$ by

$$
\left(\theta_{1}, \theta_{2}\right)=\int_{\mathbb{C}^{n} / \Lambda} \theta_{1}(w) \overline{\theta_{2}(w)} d \mu(w)
$$

with $\Delta=\operatorname{det}(H)$ and $d \mu(z)=\Delta e^{-\pi H(z, z)} d z$ as in the previous section. It is easily checked that the inner product is well-defined and $\mathcal{N}_{H, K}$ is a finite-dimensional Hilbert space.

Letting $t$ be a positive real number, let $\mathcal{H}_{t H}$ refer to the space $\mathcal{H}_{H}$ defined in the previous section with the Hermitian form $H$ replaced by $t$ times $H$. As $\operatorname{det}(t H)=t^{n} \operatorname{det}(H)=t^{n} \Delta$, the corresponding measure $d \mu_{t}(z)$ is $t^{n} \Delta e^{-\pi t H(z, z)} d z$ and the reproducing kernel, given in Theorem 4.6, is $\Phi_{t}(z, w)=e^{\pi t H(z, w)}$.

For $t>0, \lambda \in \Lambda$, and $J$ as in (3.4), we define

$$
\begin{aligned}
\Phi_{t}^{\theta, \lambda}(z, w) & =\Phi_{t}(z, w+\lambda) J(w, \lambda) e^{-\pi t H(w+\lambda, w+\lambda)+\pi t H(w, w)} \\
\Phi_{t}^{\theta}(z, w) & =\sum_{\lambda \in \Lambda} \Phi_{t}^{\theta, \lambda}(z, w)
\end{aligned}
$$

and

$$
\Phi^{\theta, \lambda}(z, w)=\Phi_{1}^{\theta, \lambda}(z, w) \text { and } \Phi^{\theta}(z, w)=\Phi_{1}^{\theta}(z, w)
$$


Using the fact that $H$ is linear in the first-component and conjugate-linear in the second, we may simplify $\Phi^{\theta, \lambda}(z, w)$ to obtain

$$
\Phi^{\theta, \lambda}(z, w)=\exp \left[\pi H(z, w+\lambda)-\frac{\pi}{2} H(\lambda, \lambda)-\pi H(\lambda, w)+2 \pi i K(\lambda)\right] .
$$

Theorem 5.1. $\Phi^{\theta}$ as in (5.1) is the reproducing kernel for $\mathcal{N}_{H, K}$.

Proof. We must verify the three properties of the reproducing kernel given in Section 2 We first check property (2), which follows from the following stronger statement: for $t \geq 1$ and $\theta \in \mathcal{N}_{H, K}$

$$
\theta(z)=\int_{\mathbb{C}^{n} / \Lambda} \Phi_{t}^{\theta}(z, w) \theta(w) d \mu(w) .
$$

Showing that the integrand is periodic is well-defined is a routine calculation whose proof we suppress. Since $\theta$ is an entire theta function, according to Theorem 3.3 , there exists a constant $C$ such that $|\theta(z)|^{2} e^{-\pi H(z, z)}<C$ for all $z \in \mathbb{C}^{n}$. Thus,

$$
\|\theta\|_{t}=\int_{\mathbb{C}^{n}}|\theta(z)|^{2} d \mu_{t}(z)<t^{n} \Delta \int_{\mathbb{C}^{n}} C e^{(1-t) \pi H(z, z)} d z .
$$

Suppose first that $t>1$. Since $H$ is positive definite, the integral above converges, and so $\theta(z) \in \mathcal{H}_{t H}$. Using the reproducing kernel for $\mathcal{H}_{t H}$ (Theorem 4.6) and the theta multiplier (3.3),

$$
\theta(z)=\int_{\mathbb{C}^{n}} \Phi_{t}(z, w) \theta(w) d \mu_{t}(w)=\int_{\mathbb{C}^{n} / \Lambda} \sum_{\lambda \in \Lambda} \Phi^{\theta, \lambda}(z, w+\lambda) \theta(w+\lambda) d \mu(w+\lambda)
$$

which gives (5.3) when $t>1$.

Now consider the case when $t=1$. We have

$$
\begin{aligned}
\Phi^{\theta}(z, & w) \theta(w) e^{-\pi H(w, w)} \\
& =\sum_{\lambda \in \Lambda} \Phi(z, w+\lambda) J(w, \lambda) e^{-\pi H(w+\lambda, w+\lambda)+\pi H(w, w)} \theta(w) e^{-\pi H(w, w)} \\
& =e^{-\pi H(z, w)} \theta(w) e^{-\pi H(w, w)} \sum_{\lambda \in \Lambda} e^{-\frac{\pi}{2} H(\lambda, \lambda)+\text { terms linear in } \lambda .}
\end{aligned}
$$

By assumption, $H$ is positive definite, and so this summation is uniformly convergent on compact sets. Of course $\mathbb{C}^{n} / \Lambda$ is compact and therefore, as $t \rightarrow 1$ from above,

$$
\int_{\mathbb{C}^{n} / \Lambda} \Phi_{t}^{\theta}(z, w) \theta(w) d \mu_{t}(w) \rightarrow \int_{\mathbb{C}^{n} / \Lambda} \Phi^{\theta}(z, w) \theta(w) d \mu(w),
$$

establishing (15.3) when $t=1$.

Next we check property (3), i.e., that

$$
\Phi^{\theta}(z, w)=\overline{\Phi^{\theta}(w, z)} .
$$

Using the representation of $\Phi^{\theta, \lambda}$ as in (5.2) together with the facts that $H$ is Hermitian and $K$ is linear and real-valued, one easily checks that $\Phi^{\theta, \lambda}(z, w)=\overline{\Phi^{\theta,-\lambda}(w, z)}$, and adding up over all $\lambda \in \Lambda$, we get the desired result.

Finally, we check that for any fixed $w \in \mathbb{C}^{n}$, the function $f(z)=\Phi^{\theta}(z, w)$ is a normalized entire theta function of $(H, K)$ type, i.e., $f \in \mathcal{N}_{H, K}$. In other words, 
we must show that $\Phi^{\theta}(z+\nu, w)=\Phi^{\theta}(z, w) J(z, \nu)$. Using identities (3.1) and (3.3.3) together with the facts that $H$ is Hermitian and $K$ is linear and real-valued,

$$
\Phi^{\theta, \lambda}(z, w+\nu)=\Phi^{\theta, \lambda+\nu}(z, w) \overline{J(z, \nu)} .
$$

Adding up over all $\lambda \in \Lambda$ and using (5.4), we obtain the desired result.

Thus, $\Phi^{\theta}$ satisfies all three properties of being a reproducing kernel, completing the proof.

Theorem 5.2. $\operatorname{dim} \mathcal{N}_{H, K}=\operatorname{det}(H) \operatorname{Vol}\left(\mathbb{C}^{n} / \Lambda\right)$.

Proof. Expanding $\Phi^{\theta}$ as in (5.1), $J$ as in (3.3) and letting $S_{\lambda}(z)=e^{\pi(H(z, \lambda)-H(\lambda, z))}$, Theorem 2.1 shows that

$$
\operatorname{dim} \mathcal{N}_{H, K}=\int_{\mathbb{C}^{n} / \Lambda} \Phi^{\theta}(z, z) d \mu(z)=\Delta \sum_{\lambda \in \Lambda} e^{-\pi H(\lambda, \lambda)+2 \pi i K(\lambda)} \int_{\mathbb{C}^{n} / \Lambda} S_{\lambda}(z) d z .
$$

Since we are considering only normalized theta functions, $H(z, w)=2 i L(z, w)$, and so $S_{\lambda}(z)=e^{2 \pi i(L(z, \lambda)-L(\lambda, z))}$. Clearly $S_{\lambda}(z+w)=S_{\lambda}(z) S_{\lambda}(w)$. Moreover, by Theorem 3.2, for $\nu \in \Lambda, L(\nu, \lambda)-L(\lambda, \nu)=E(\nu, \lambda)$ is an integer, and so $S_{\lambda}(\nu)=1$ and $S_{\lambda}(z+\nu)=S_{\lambda}(z)$. Thus, $S_{\lambda}$ is a group character on $\mathbb{C}^{n} / \Lambda$, which means that the integral is zero unless $S_{\lambda}$ is identically 1 . Therefore, the only surviving term in the summation is the one with $\lambda=0$, which gives

$$
\operatorname{dim} \mathcal{N}_{H, K}=\Delta \int_{\mathbb{C}^{n} / \Lambda} 1 d z=\Delta \operatorname{Vol}\left(\mathbb{C}^{n} / \Lambda\right)=\operatorname{det}(H) \operatorname{Vol}\left(\mathbb{C}^{n} / \Lambda\right) .
$$

Theorem 5.3. If $H$ is associated to a nondegenerate Riemann form E with Pfaffian $P f$, then $\operatorname{dim} \mathcal{N}_{H, K}=P f$.

Proof. For $z, w \in \mathbb{C}^{n}$, let us write $z=x+i y$ and $w=u+i v$ where $u, v, x, y$ are all real. By the Spectral Theorem, $H=U^{*} D U$ for some unitary matrix $U$ and positive real diagonal matrix $D$. Write $U=A+i B$ with $A$ and $B$ real. Then, considering $\mathbb{C}^{n}$ as a complex vector space ( $n$ ot as an $\mathbb{R}$-module over a $\mathbb{Z}$-basis for $\Lambda$ ), we have (by Theorem 3.2 (

$$
\begin{aligned}
E(z, w)=\operatorname{Im}(H(z, w)) & =\operatorname{Im}\left((u+i v)^{*}(A+i B)^{*} D(A+i B)(x+i y)\right) \\
& =\left[\begin{array}{l}
u \\
v
\end{array}\right]^{T}\left[\begin{array}{cc}
A & -B \\
B & A
\end{array}\right]^{T}\left[\begin{array}{cc}
0 & -D \\
D & 0
\end{array}\right]\left[\begin{array}{cc}
A & -B \\
B & A
\end{array}\right]\left[\begin{array}{l}
x \\
y
\end{array}\right] .
\end{aligned}
$$

Suppose that $\Lambda$ is spanned over $\mathbb{Z}$ by $\left\{\tau_{1}, \ldots, \tau_{2 n}\right\}$, let $R+i S$ be the $n \times 2 n$ matrix whose $j$ th column is $\tau_{j}$. Thus, on this basis we have

$$
E(a, b)=b^{T}\left[\begin{array}{c}
R \\
S
\end{array}\right]^{T}\left[\begin{array}{cc}
A & -B \\
B & A
\end{array}\right]^{T}\left[\begin{array}{cc}
0 & D \\
-D & 0
\end{array}\right]\left[\begin{array}{cc}
A & -B \\
B & A
\end{array}\right]\left[\begin{array}{c}
R \\
S
\end{array}\right] a
$$

It is clear that

$$
\left|\operatorname{det}\left[\begin{array}{l}
R \\
S
\end{array}\right]\right|=\operatorname{Vol}\left(\mathbb{C}^{n} / \Lambda\right)
$$

On the other hand, since $U=A+i B \rightarrow \operatorname{det}\left[\begin{array}{cc}A & -B \\ B & A\end{array}\right]$ is a continuous homomorphism of the connected compact group of $n \times n$ unitary matrices into $\mathbb{R}^{\times}$, its image 
is constant; that is,

$$
\operatorname{det}\left[\begin{array}{cc}
A & -B \\
B & A
\end{array}\right]=1
$$

Thus, by Theorem 5.2,

$$
P f=\sqrt{\operatorname{det}(E)}=\operatorname{det}(D) \operatorname{Vol}\left(\mathbb{C}^{n} / \Lambda\right)=\operatorname{det}(H) \operatorname{Vol}\left(\mathbb{C}^{n} / \Lambda\right) .
$$

\section{REFERENCES}

1. V. Bargman, On a Hilbert space of analytic functions and an associated integral transform, Comm. Pure Appl. Math. 14 (1961) 187-214.

2. P. Cartier, Quantum mechanical commutation relations and theta functions, in Algebraic Groups and Discontinuous Subgroups, AMS Proc. Sympos. Pure Math. 9 (1966), 361-383. MR 35:7654

3. M. Eichler, Eine Verallgemeinerung der Abelschen Integrale, Math. Zeit. 67 (1957), 267-298. MR 19:740a

4. G. Frobenius, Über die Grundlagen der Theorie der Jacobischen Functionen, J. Reine Angew. Math. 97 (1884), 188-223.

5. D. Hejhal, Kernel functions, Poincaré series and LVA, in In the tradition of Ahlfors and Bers, I. Kra and B. Maskit (ed.), Contemporary Mathematics 256 (2000), 173-201. MR 2001e:11042

6. N. Hurt, Geometric quantization in action, Reidel Publishing (1983). MR 84f:58053

7. J. Igusa, Theta Functions, Springer-Verlag, New York, 1972. MR 48:3972

8. S. Lang, Introduction to Algebraic and Abelian Functions, Second Ed., Springer-Verlag, New York, 1982. MR 84m:14032

9. D. Newman and H. Shapiro, Certain Hilbert spaces of entire functions, Bull. Amer. Math. Soc. 72 (1966) 971-977. MR 34:4890

10. V. Murty, Introduction to Abelian Varieties, American Mathematical Society, Providence, 1993. MR 94h:14045

11. I. Satake, Fock representations and theta-functions, in Advances in the Theory of Riemann Surfaces, Ann. of Math. Studies 66, Princeton (1971), 393-405. MR 54:12657]

12. A. Selberg, Automorphic functions and integral operators, in Seminars on Analytic Functions II, Institute for Advanced Study (1957), 152-161. Article 28 in Selberg, Collected Works, Springer Verlag (1989). MR 92h:01083

13. H. Shimizu, On Discontinuous Groups Operating on the Product of the Upper Half Planes, Annals of Math., Vol. 77, pp. 33-71, 1963. MR 26:2641

14. H. Swinnerton-Dyer, Analytic Theory of Abelian Varieties, Cambridge University, Cambridge, 1974. MR 51:3180

15. A. Weil, Sur certains groupes d'opérateurs unitaires, Acta Math. 111 (1964), 143-211, Collected Works, vol. 3. MR 29:2324

Department of Mathematics, Stanford University, Stanford, California 94305

E-mail address: bump@math.stanford.edu

Department of Mathematics, Stanford University, Stanford, California 94305

E-mail address: apekker@stanfordalumni.org

Current address: 1841 Palisades Drive, Santa Rosa, California 95403 